\title{
GATE: a simulation toolkit for PET and SPECT
}

\author{
S Jan ${ }^{1}$, G Santin ${ }^{2,24}$, D Strul ${ }^{2,25}$, S Staelens ${ }^{3}$, K Assié ${ }^{4}$, D Autret ${ }^{5}$, \\ S Avner ${ }^{6}$, R Barbier $^{7}$, M Bardiès $^{5}$, P M Bloomfield ${ }^{8}$, D Brasse ${ }^{6}$, \\ V Breton ${ }^{9}$, P Bruyndonckx ${ }^{10}$, I Buvat ${ }^{4}$, A F Chatziioannou ${ }^{11}$, Y Choi ${ }^{12}$, \\ Y H Chung ${ }^{12}$, C Comtat ${ }^{1}$, D Donnarieix ${ }^{9,13}$, L Ferrer ${ }^{5}$, S J Glick ${ }^{14}$, \\ C J Groiselle ${ }^{14}$, D Guez ${ }^{15}$, P-F Honore ${ }^{15}$, S Kerhoas-Cavata ${ }^{15}$, \\ A S Kirov ${ }^{16}$, V Kohli ${ }^{11}$, M Koole ${ }^{3}$, M Krieguer ${ }^{10}$, D J van der Laan ${ }^{17}$, \\ F Lamare $^{18}$, G Largeron ${ }^{7}$, C Lartizien ${ }^{19}$, D Lazaro ${ }^{9}$, M C Maas $^{17}$, \\ L Maigne ${ }^{9}$, F Mayet $^{20}$, F Melot ${ }^{20}$, C Merheb $^{15}$, E Pennacchio ${ }^{7}$, J Perez $^{21}$, \\ U Pietrzyk $^{21}$, F R Rannou ${ }^{11,22}$, M Rey ${ }^{2}$, D R Schaart ${ }^{17}$, C R Schmidtlein ${ }^{16}$, \\ L Simon $^{2,26}$, T Y Song ${ }^{12}$, J-M Vieira ${ }^{2}$, D Visvikis ${ }^{18}$, R Van de Walle ${ }^{3}$, \\ E Wieërs ${ }^{10,23}$ and C Morel $^{2}$ \\ ${ }^{1}$ Service Hospitalier Frédéric Joliot (SHFJ), CEA, F-91401 Orsay, France \\ 2 LPHE, Swiss Federal Institute of Technology (EPFL), CH-1015 Lausanne, Switzerland \\ 3 ELIS, Ghent University, B-9000 Ghent, Belgium \\ ${ }^{4}$ INSERM U494, CHU Pitié-Salpêtrière, F-75634 Paris, France \\ 5 INSERM U601, CHU Nantes, F-44093 Nantes, France \\ ${ }^{6}$ Institut de Recherches Subatomiques, CNRS/IN2P3 et Université Louis Pasteur, \\ F-67037 Strasbourg, France \\ ${ }^{7}$ Institut de Physique Nucléaire de Lyon, CNRS/IN2P3 et Université Claude Bernard, \\ F-69622 Villeurbanne, France \\ ${ }^{8}$ PET Group, Centre for Addiction and Mental Health, Toronto, Ontario M5T 1R8, Canada \\ ${ }^{9}$ Laboratoire de Physique Corpusculaire, CNRS/IN2P3, Université Blaise Pascal, \\ Campus des Cézeaux, F-63177 Aubière, France \\ ${ }^{10}$ Inter-University Institute for High Energies, Vrije Universiteit Brussel, B-1050 Brussel, \\ Belgium \\ ${ }^{11}$ Crump Institute for Molecular Imaging, University of California, Los Angeles, \\ CA 90095-1770, USA \\ 12 Department of Nuclear Medicine, Samsung Medical Center, Sungkyunkwan University School \\ of Medicine, Seoul 135-710, Korea \\ ${ }^{13}$ Département de Curiethérapie-Radiothérapie, Centre Jean Perrin, F-63000 Clermont-Ferrand, \\ France \\ ${ }^{14}$ University of Massachusetts Medical School, Division of Nuclear Medicine, Worcester, \\ MA 01655, USA \\ 15 DAPNIA, CEA Saclay, F-91191 Gif-Sur-Yvette, France \\ ${ }^{16}$ Department of Medical Physics, Memorial Sloan-Kettering Cancer Center, New York, \\ NY 10021, USA \\ ${ }^{17}$ Delft University of Technology, IRI, Radiation Technology, 2629 JB Delft, \\ The Netherlands \\ 18 INSERM U650, Laboratoire de Traitement de l'Information Médicale (LATIM), \\ CHU Morvan, F-29609 Brest, France \\ 19 ANIMAGE-CERMEP, Université Claude Bernard Lyon 1, F-69003 Lyon, France \\ ${ }^{20}$ Laboratoire de Physique Subatomique et de Cosmologie, CNRS/IN2P3 et Université \\ Joseph Fourier, F-38026 Grenoble, France
}

\footnotetext{
${ }^{24}$ Present address: ESA-European Space and Technology Centre, 2200 AG Noordwijk, The Netherlands.

${ }^{25}$ Present address: CNRS-LESIA, Observatoire de Meudon, F-92190 Meudon, France.

${ }^{26}$ Present address: Institut Curie, Service de Physique Médicale, F-75005 Paris, France. 
${ }^{21}$ Institute of Medicine, Forschungszemtrum Juelich, D-52425 Juelich, Germany

22 Departamento de Ingenieria Informatica, Universidad de Santiago de Chile, Santiago, Chile

${ }^{23}$ Nucleair Technologisch Centrum, Dept. Industriële Wetenschappen en Technologie,

Hogeschool Limburg, B-3590 Diepenbeek, Belgium

E-mail: christian.morel@epfl.ch

Received 14 May 2004, in final form 26 July 2004

Published 10 September 2004

Online at stacks.iop.org/PMB/49/4543

doi:10.1088/0031-9155/49/19/007

\begin{abstract}
Monte Carlo simulation is an essential tool in emission tomography that can assist in the design of new medical imaging devices, the optimization of acquisition protocols and the development or assessment of image reconstruction algorithms and correction techniques. GATE, the Geant4 Application for Tomographic Emission, encapsulates the Geant4 libraries to achieve a modular, versatile, scripted simulation toolkit adapted to the field of nuclear medicine. In particular, GATE allows the description of time-dependent phenomena such as source or detector movement, and source decay kinetics. This feature makes it possible to simulate time curves under realistic acquisition conditions and to test dynamic reconstruction algorithms. This paper gives a detailed description of the design and development of GATE by the OpenGATE collaboration, whose continuing objective is to improve, document and validate GATE by simulating commercially available imaging systems for PET and SPECT. Large effort is also invested in the ability and the flexibility to model novel detection systems or systems still under design. A public release of GATE licensed under the GNU Lesser General Public License can be downloaded at http:/www-lphe.epfl.ch/GATE/. Two benchmarks developed for PET and SPECT to test the installation of GATE and to serve as a tutorial for the users are presented. Extensive validation of the GATE simulation platform has been started, comparing simulations and measurements on commercially available acquisition systems. References to those results are listed. The future prospects towards the gridification of GATE and its extension to other domains such as dosimetry are also discussed.
\end{abstract}

\title{
1. Introduction
}

Emission tomography is becoming increasingly important in modern medicine for both diagnostic and treatment monitoring, with a demand for higher imaging quality, accuracy and speed. Recently enhanced by the wider availability of powerful computer clusters, Monte Carlo simulations have become an essential tool for current and future emission tomography development. Examples of research areas benefiting from these developments are the design of new medical imaging devices, the optimization of acquisition protocols and the development and assessment of image reconstruction algorithms and correction techniques.

Currently there are numerous Monte Carlo simulation packages for either positron emission tomography (PET) or single photon emission computer tomography (SPECT), each 
with different advantages disadvantages and levels of reliability (Buvat and Castiglioni 2002). Accurate and versatile general-purpose simulation packages such as Geant3 (Brun et al 1987), EGS4 (Bielajew et al 1994), MCNP (Briesmeister 1993) and most recently Geant4 (Agostinelli et al 2003) are available. These packages include well-validated physics models, geometry modelling tools and efficient visualization utilities. However, it is quite difficult to tailor these packages to PET and SPECT. On the other hand, the dedicated Monte Carlo codes developed for PET and SPECT suffer from a variety of drawbacks and limitations in terms of validation, accuracy and support (Buvat and Castiglioni 2002). While an adaptation of EGS4 to radiation therapy applications exists (Rogers et al 1995, Kawrakov and Rogers 2003), there are no dedicated PET or SPECT Monte Carlo programmes that are detailed and flexible enough for realistic simulations of emission tomography detector geometries. SimSET (Harrison et al 1993), one of the most powerful dedicated codes enabling PET and SPECT simulations, enables a precise and efficient modelling of physics phenomena and basic detector designs (e.g. ring detectors and planar detectors), but it has limitations with respect to the range of detector geometries that can be modelled. For example, a detector ring cannot be subdivided into individual crystals and the gaps between the crystals and the grouping of crystals into blocks cannot be modelled. In addition, neither SimSET, nor any other publicly available codes account for time explicitly, which limits their use for modelling time-dependent processes such as tracer kinetics or bed motion.

Clearly, a Monte Carlo code capable of accommodating complex scanner geometry and imaging configurations in a user-friendly way, while retaining the comprehensive physics modelling abilities of the general purpose codes is needed. Furthermore, the need is to have a platform that can model decay kinetics, deadtime and movement, while benefitting from the same versatility and support as that of the general-purpose simulation codes. Object-oriented technology appeared to be the best choice to ensure high modularity and re-usability for a PET and SPECT simulation tools. Therefore, we selected the simulation toolkit developed in C++ by the Geant 4 collaboration (Agostinelli et al 2003), and decided to foster long-term support and maintenance by sharing code development among many research groups forming the OpenGATE collaboration.

This paper presents a detailed description of the design and development of a Monte Carlo tool by the OpenGATE collaboration which satisfies the requirements mentioned above. It was launched at first (Strul 2001a, 2001b) as an aid in the design of the ClearPET prototype scanners being developed by the Crystal Clear collaboration (Ziemons et al 2003). GATE, the Geant4 Application for Tomographic Emission (Santin et al 2003, Strul et al 2003, Assié et al 2004a), incorporates the Geant4 libraries in a modular, versatile and scripted simulation toolkit that is specifically adapted to the field of nuclear medicine. A public release of GATE licensed under the GNU Lesser General Public License (LGPL 1999) can be downloaded at http://www-lphe.epfl.ch/GATE/.

\section{GATE basics}

GATE was designed with several objectives in mind. First, the use of the GATE software should not require any knowledge of $\mathrm{C}++$. End-users from the nuclear medicine community should be able to use GATE without worrying about the programming details. Second, as many nuclear medicine diagnostic techniques share similar concepts, GATE software components should be general enough to be reused from one context to another. Last, GATE should be modular, and thus be able to evolve as new applications are envisioned. 


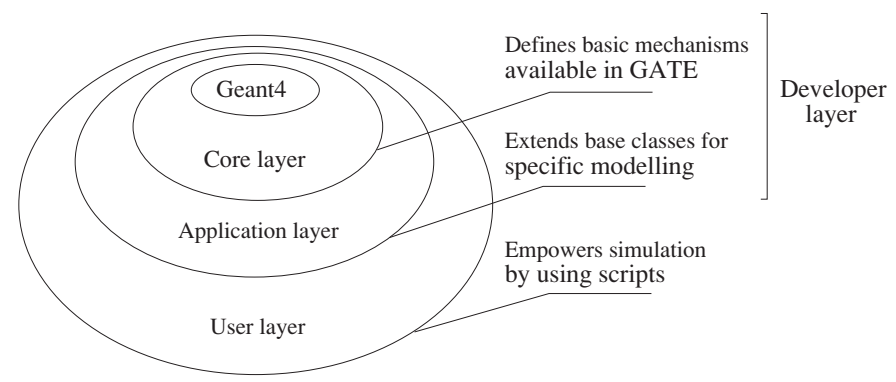

Figure 1. Sketch of the layered architecture of GATE.

\subsection{Architecture}

The requirements discussed above are met using a layered architecture sketched in figure 1 . The core of GATE, developed in $\mathrm{C}++$, defines the main tools and features of GATE. The application layer is an extensible set of $\mathrm{C}++$ classes based on the GATE core. On top of the application layer is the user layer, where end-users can simulate experiments using an extended version of the Geant4 scripting language.

The GATE developer layer consists of the core layer and the application layer. It is built from the various classes that provide the most general features of GATE. These classes define which tools are available, what developers can do and how they can do it. The core layer includes some base classes that are common or even mandatory in all Geant4based simulations, such as those involved in the construction of the geometry, the interaction physics, the event generation and the visualization management. In addition, the core layer includes classes that are specific to GATE simulations, such as the GATE virtual clock for time management. Thus, the core layer defines the basic mechanisms available in GATE for geometry definition, time management, source definition, detector electronics modelling and data output.

The application layer is composed of classes derived from the base classes of the core layer to model specific objects or processes. For example, the core layer defines the base class for volumes, and the application layer comprises all the derived classes for modelling specific volumes, including boxes, spheres, cylinders and trapezoids. Similarly, the application layer includes all the specific movement models derived from the movement base class, including translations, rotations, orbits and oscillations. Thus, the range of features available in GATE can increase as new application classes are developed, while the general structure remains unaffected.

In the user layer, Geant4 provides mechanisms for running simulations both interactively or batch-wise using scripts. An important principle of GATE is that each class must provide dedicated extensions to the command interpreter class, so that the functionality provided by the class is available through script commands. The end-users of GATE therefore do not have to perform any $\mathrm{C}++$ coding. The complete set-up of a nuclear medicine experiment can be easily defined using the script language, as shown in figure 2.

\subsection{Systems}

When defining the geometry for a tomograph, specific guidelines with respect to the geometrical hierarchy of the tomograph components must be followed, so that the Geant4 particles interaction histories, called hits, occurring in the detector, can be processed to 


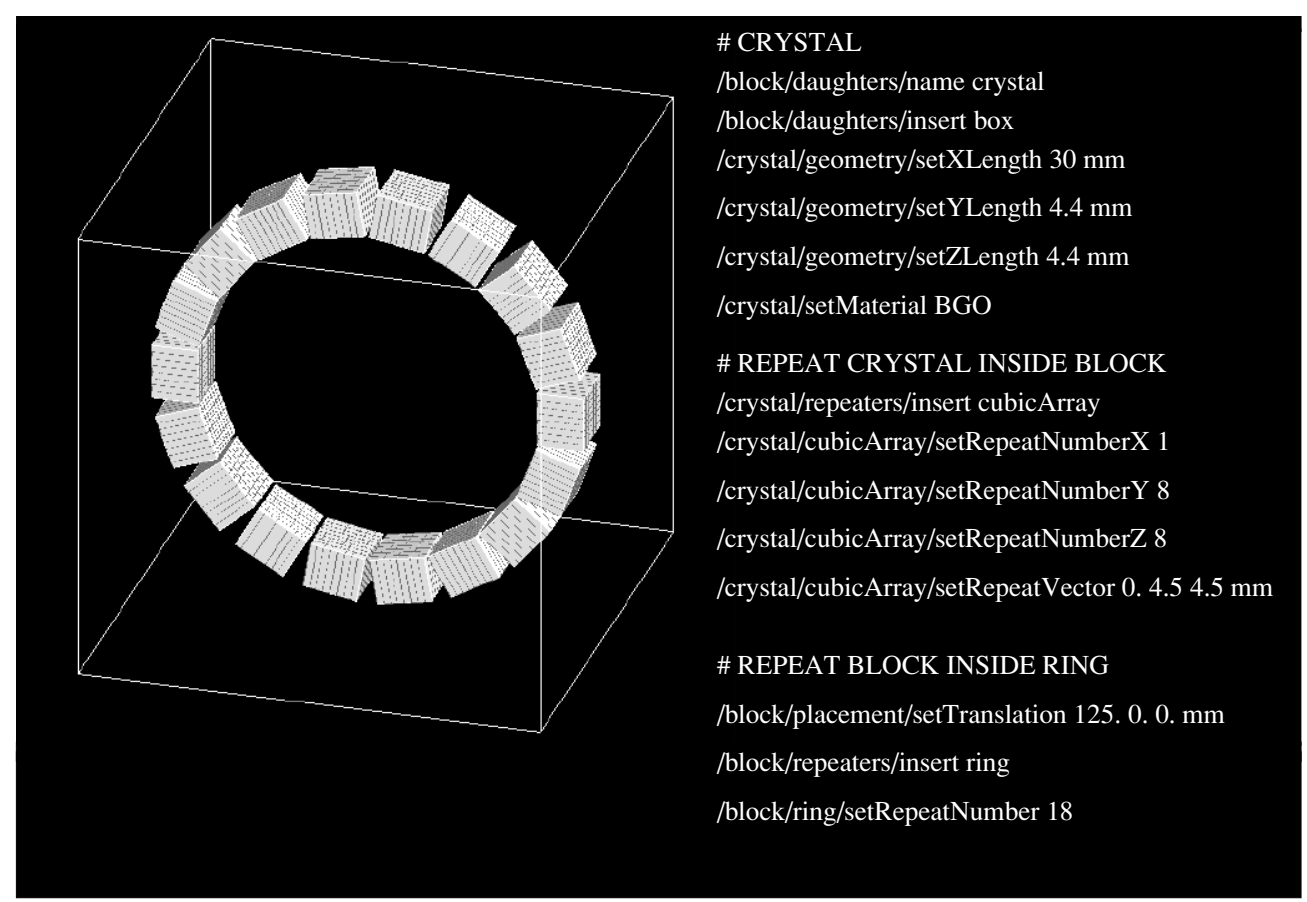

Figure 2. Ring detector geometry obtained by using the command scripts displayed in the figure. This script models a detector-block, where $4.4 \times 4.4 \mathrm{~mm}$ BGO crystals of $30 \mathrm{~mm}$ thickness are repeated in an $8 \times 8$ matrix. A cubic array repeater command produces an $8 \times 8$ cubic array of crystals from a single crystal with a crystal picth of $4.5 \mathrm{~mm}$ in both directions. A ring repeater places the block at $125.0 \mathrm{~mm}$ from the origin on the $x$-axis and replicates it 18 times about a cylinder around the $Z$-axis.

realistically mimic detector output. Most PET scanners are built following comparable concepts: one or more rings, each ring consisting of several scintillator blocks, each block being subdivided in crystal pixels. For SPECT, similar concepts exist: a gamma camera with a continuous or pixelated crystal, and a collimator. Most of these geometrical concepts are common to many different imaging systems. To facilitate the hierarchical description of a tomograph, predefined global systems are used. A system is defined as a family of geometries compatible with one or several data output formats. The main property of a system is that its geometry description is supported by specific list-mode or histogrammed (sinogram or projection) data output formats. Currently there are five systems available in GATE: one for SPECT, three for PET - two for block detector geometries and one for continuous pixellated geometries - and a generic system appropriate to model novel tomographic paradigms. This latter system is completely open and provides only basic building blocks for the definition of a tomographic experiment.

\subsection{Management of time and movements}

One of the distinctive features of GATE is the management of time-dependent phenomena (Santin et al 2003, Strul et al 2003). The synchronization of the source kinetics with the movement of the geometry thus allows for the simulation of realistic acquisition conditions including patient movement, respiratory and cardiac motions, scanner rotation or changes 
in activity distribution over time. Dealing with time in GATE includes: (a) defining the movements associated with the physical volumes that describe the detector and phantom; (b) describing the radioactive sources and (c) specifying the start and stop times of the acquisition (which are equivalent to the start and stop times in a real experiment).

The Geant 4 geometry architecture requires the geometry to be static during a simulation. However, the typical duration of a single event is very short when compared to movements in the geometry model or bio-kinetics. Movements are synchronized with the evolution of the source activities by subdividing the acquisition time frames (typically of the order of minutes or hours) into smaller time steps. At the beginning of each time step, the geometry is updated according to the requested movements. During each time step, the geometry is held at rest and the simulation of the particle transport proceeds. Within the time steps, the source is allowed to decay so that the number of events decreases exponentially from one time step to the next, and within the time steps themselves. The proper timing of the simulated event sequence is a key feature for modelling time-dependent processes such as count rates, random coincidences, event pile-up and detector deadtime (Simon et al 2004). Between time steps, the position and the orientation of a subset of daughter volumes can be changed to mimic a movement such as a rotation or a translation. These displacements are parametrized by their velocity. It is the responsibility of the user to set the time step duration short enough to produce smooth changes. Combinations of translations and rotations allow the simulation of complex acquisition trajectories of the detectors such as parametrized eccentric rotations.

Geant 4 does not allow the movement of sources. Therefore, in GATE, an emission volume is defined so that it encompasses the actual source's volume throughout its range of displacement. To enable movement of the activity distribution, an additional volume is defined to confine the emission within the intersection of the emission and the confinement volumes. This confinement volume defines the shape and size of the actual source and moves within the emission volume.

\section{Physics}

\subsection{Radioactive sources}

A source in GATE is defined by its particle type (e.g. radionuclide, gamma, positrons, etc), position (volume), direction (solid angle), energy (spectrum) and activity. The lifetime of a radioactive source is usually obtained from the Geant 4 database, but it can also be set by the user to approximate a decay source through the emission of its decay products (e.g. positrons or gammas).

The activity determines the decay rate for a given source during the simulated acquisition time. Radioactive decay of radionuclides with secondary particle emission is performed by the Geant4 radioactive decay module (RDM), which has been modified so that GATE source manager maintains control over the definition of decay time. Continuous event time flow is obtained by using a virtual clock that defines an absolute time $t$ used to initialize the Geant 4 internal tracking time. Random time intervals $\delta t$ between events that occur at time $t$ are sampled from an exponential distribution:

$$
p(\delta t)=A(t) \exp (-A(t) \delta t)
$$

where $A(t)=A_{0} \exp \left(\left(t-t_{0}\right) / \tau\right)$ is the source activity at time $t, A_{0}$ is the user defined source activity at time $t_{0}$ and $\tau$ is the lifetime. When the resulting decay time exceeds the end of the current time step, the run is terminated and a new one is started, allowing for the synchronization of the sources with the geometry movements. Multiple sources can be defined 
with independent properties. For each event, a proposed time interval is sampled for each source according to equation (1), and the shortest one is chosen for the primary generation. The overall behaviour of this mechanism is such that, at all times, the relative importance of each source is proportional to its activity, while the overall time interval sampling is determined by the total activity of all sources. Voxelized phantom or patient data can be used as sources to reproduce realistic acquisitions: emission data are converted into activity levels, and GATE can read in voxelized attenuation map and converts the grey scale into material definitions using an analogous translator.

\subsection{Positron emission}

GATE includes two modules dedicated to PET (Jan 2002). The first uses the von Neumann algorithm (von Neumann 1951) to randomly generate the positron energy according to the measured $\beta^{+}$spectra. This method greatly increases the speed of the simulation by bypassing the decay of radionuclides process used by Geant 4 . The $\beta^{+}$spectra of three commonly used radionuclides $\left({ }^{11} \mathrm{C},{ }^{15} \mathrm{O}\right.$ and $\left.{ }^{18} \mathrm{~F}\right)$ have been parametrized in GATE according to the Landolt-Börnstein tables (Behrens and Janecke 1969).

The second module deals with the acollinearity of the two annihilation photons, which is not accounted for in Geant 4 . In GATE, acollinearity is modelled using a $0.58^{\circ}$ full width at half maximum (FWHM) Gaussian blur. This width corresponds to experimental values measured in water (Iwata et al 1997).

\subsection{Interaction modelling with standard energy and low energy packages}

A material database file contains all material parameters required by Geant 4 to calculate the interaction cross-sections and is easily modified by the user. In contrast to Geant4, GATE only uses natural isotopic abundances. The fact that these cannot be modified by the user has little bearing on GATE applications since isotopic abundances are unimportant in low to mid-energy photon and charged particle interactions. The electromagnetic interactions used in GATE are derived from Geant 4 . The electromagnetic physics package manages electrons, positrons, $\gamma$-rays, $\mathrm{x}$-rays, optical photons, muons, hadrons and nuclei. As in Geant4, GATE can use two different packages to simulate electromagnetic processes: the standard energy package, and the low energy package. In the standard energy package, photoelectric effect and Compton scatter can be simulated at energies above $10 \mathrm{keV}$. Under 100, $\mathrm{keV}$ however, relative errors on the cross-sections are higher than 10\% and can rise up above 50\% (Jan 2002, Lazaro 2003). The low energy package models photon and electron interactions down to $250 \mathrm{eV}$ and includes Rayleigh scattering. For biomedical applications, it provides more accurate models for the electromagnetic interactions. However, this comes at the price of increased computing time.

\subsection{Secondary production cuts}

GATE inherits the Geant 4 capability to set thresholds for the production of secondary electrons, $\mathrm{x}$-rays and delta-rays (Agostinelli et al 2003). In biomedical applications, eliminating the secondary particles whose initial energy is below the production threshold increases the computing efficiency.

Because low energy processes generate more secondary particles than standard energy processes, cuts affect simulation speed more strongly when applied with the low energy package. Turning off the production of electrons, x-rays and delta-rays by setting high 


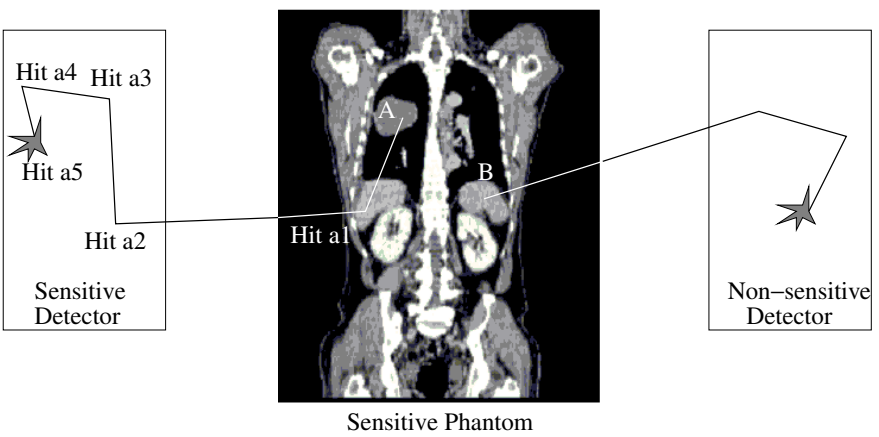

Figure 3. Particle interactions in a crystalSD attached to a scintillator block and a phantomSD attached to a volume filled with tissue material. The trajectory of particle A shows 1 hit in the phantomSD (Hit a1) and 4 hits in the crystalSD (Hit a2 to Hit a5). Particle B does not interact within a sensitive volume, thus no hit information is stored.

thresholds may result in a substantial increase in computing speed for a typical simulation of a PET scanner. In many cases, the accuracy of the simulation at the level of single or coincidence photon counting is preserved.

\section{Digitization}

Digitization is the process of simulating the electronics response of a detector within a scanner. This involves the conversion of the charged particle and photon interactions into energy bins, detection positions and coincidences. To do this, portions of the scanner geometry are designated as sensitive detectors, which record interactions within these regions. The digitizer chain then processes these recorded interactions and produces counts and coincidences. The sensitive detectors and digitizer chain are described below.

\subsection{Sensitive detectors}

Sensitive detectors are used to store information about particle interactions (hereafter referred to as hits) within volumes. GATE only stores hits for those volumes that have a sensitive detector attached. Two types of sensitive detectors are defined in GATE: the crystal sensitive detector (crystalSD) is used to generate hits from interactions that occur inside the detector portions of the scanner (figure 3). The phantom sensitive detector (phantomSD) is used to detect and count the Compton and Rayleigh interactions occurring within the scanner's field-of-view (FOV).

\subsection{Digitizer chain}

The digitizer chain mimics a realistic detection process by building the physical observables from the hits. The observables of each detected event are energy, position and time of the interaction. The digitizer consists of a chain of processing modules that takes a list of hits from the sensitive detectors and transforms them into pulses referred to as singles. The key elements of this chain are now briefly described.

4.2.1. Hit adder. A particle entering a sensitive detector can generate multiple hits, as shown in figure 3. For instance, a gamma ray interacting within a scintillation crystal can generate 
hits corresponding to several Compton scattering events and a photoelectric absorption. The hit adder sums the deposited energy of these hits within a sensitive detector to yield a pulse. The position of the pulse is calculated from the energy-weighted centroid of the hit positions, and the time of the pulse is set to that of the first hit within the volume. If a particle interacts in several sensitive detectors the hit adder will generate a list of pulses, one for each sensitive detector.

4.2.2. Pulse reader. With the exception of one-to-one readout schemes, scanners often have a readout segmentation different from the detector segmentation. To simulate this, a pulse reader module adds the pulses together within a user-defined group of sensitive detectors. This yields a pulse containing the total energy deposited within the group of sensitive detectors. The position of this pulse is set to that of the pulse from the adder that has the largest energy (winner-takes-all paradigm).

4.2.3. User modules. Following the hit adder and the pulse reader, which regroup the hits into pulses and then sum pulses, the remaining modules of the digitizer chain transform these pulses into the physical observables of the scanner (i.e. singles). These modules are discussed below.

Energy resolution. This module applies a Gaussian blur to the energy of the pulse, $E$, with an FWHM of $R \times E$. The FWHM energy resolution, $R$, is given by

$$
R=\frac{R_{0} \sqrt{E_{0}}}{\sqrt{E}}
$$

where $R_{0}$ is the user-defined FWHM energy resolution at a given energy, $E_{0}$.

A more elaborate model propagates the relative variances of the physical processes involved with light collection and detection in a spectrometric chain as

$$
v_{E}=v_{N}+\frac{v_{\eta}}{\bar{N}}+\frac{v_{\epsilon}}{\bar{N} \eta}+\frac{v_{M}}{\bar{N} \eta \epsilon}
$$

where $v_{E}, v_{N}, v_{\eta}, v_{\epsilon}$ and $v_{M}$ are the relative variances on $E$, on the number of scintillation photons $N$, on the light collection efficiency $\eta$, on the quantum efficiency of the photo-detector $\epsilon$ and on the gain of the photo-detector $M$, respectively. Both the light collection efficiency and the quantum efficiency are binomial processes with probabilities of $\eta$ and $\epsilon$, respectively. If the scintillation process is assumed to follow a Poisson law with a mean equal to $\bar{N}=E \times L Y$, where $L Y$ is the light yield of the scintillator, then relative variance on $E$ is given by

$$
v_{E}=\frac{1+v_{M}}{\bar{N} \eta \epsilon}
$$

In the case of a photomultiplier, $v_{M}$ is approximately 0.1 and the FWHM energy resolution is estimated using the equation:

$$
R=\sqrt{2.35^{2} \frac{1.1}{\bar{N} \eta \epsilon}+R_{i}{ }^{2}}
$$

where $R_{i}$ is the intrinsic resolution of the scintillator (Kuntner et al 2002).

Energy window. Upper and lower energy thresholds can be set for several energy windows by using multiple processor chains. These thresholds are applied using either a step or sigmoid function.

Spatial resolution. For SPECT, spatial resolution is modelled using a Gaussian blur of the position. For PET, interaction position is calculated by the pulse reader which simulates 
the intrinsic spatial resolution of the detector. More elaborate models can be derived for continuous crystal detector PET systems (Staelens et al 2004a). These models are currently under development.

Time resolution. Simulation of time jitter can be obtained using a Gaussian blur of the pulse time.

Deadtime. Both paralyzable and non-paralyzable deadtimes can be modelled explicitly on an event-by-event basis. While these models represent the idealized behaviour, they correctly predict the theoretical lifetimes for both types of deadtimes (Simon et al 2004).

Coincidence. At the end of a digitizer chain, a coincidence sort can be added to find pairs of singles that are in coincidence. Pairs of singles can be considered coincidences whenever the time interval between the singles is less than a user-defined coincidence window. Each single is stored with its corresponding event number. If the event numbers of the singles associated with a coincidence are different, this is a random coincidence. A similar flag exists for Compton scattered events. The Compton scatter flag can be used to differentiate true from scattered coincidence pairs that have identical event flags. Multiple coincidences corresponding to more than two singles within the same coincidence window are discarded.

\subsection{DigiGATE}

In GATE standard operation mode, primary particles are generated by a source manager and then propagated through the attenuating geometry before generating hits in the sensitive detectors, which are then processed by the digitizer chain. While this operating mode is suitable for many purposes, it is inefficient for the optimization of the digitizer chain parameters. This is best done by comparing the results from different sets of digitizer parameters using the same series of hits. To perform this specific task, GATE offers an operating mode named DigiGATE. In this mode, hits are read from a data file generated by GATE and fed directly into the digitizer chain. The same command scripts are used for both the hit generation simulation and DigiGATE simulations. Thus, all conditions are kept identical in the simulations including time dependence.

\section{Simulation benchmarks}

Two benchmarks, one for PET and one for SPECT, are included in the GATE distribution. These benchmarks check the integrity of the installation or upgrade, and also allow for the comparison of CPU performance on different computing platforms. In addition, they provide examples of how to use the main features of GATE to simulate PET or SPECT experiments. Furthermore, they serve as examples on how to analyse output. Each benchmark consists of macros to run the simulation, analyse simulation output and generate figures. In addition, a set of baseline figures are included for a comparison of the user's results with those from a correct run.

\subsection{PET benchmark}

The PET benchmark (figure 4) simulates a whole-body scanner that does not correspond to any existing system. Rather, it serves as a simple system that contains the majority of GATE features. It consists of eight detector heads arranged within a $88 \mathrm{~cm}$ diameter by $40 \mathrm{~cm}$ axial length octagonal cylinder. Each head is made of 400 detector blocks and each block is a $5 \times 5$ array of dual-layer LSO-BGO crystals. The heads are equipped with partial septa that rotate 


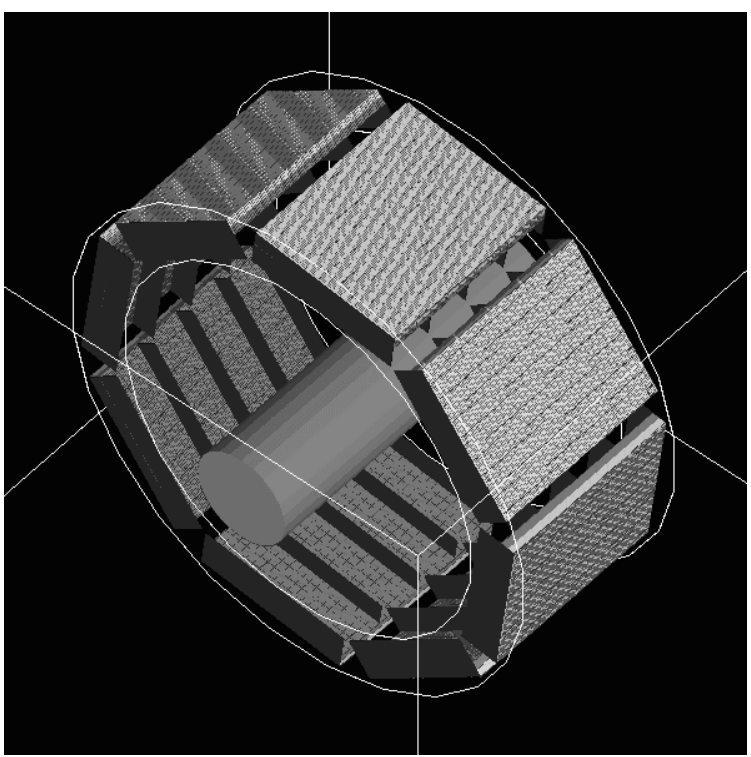

Figure 4. Illustration of the PET benchmark set-up.

in a step-and-shoot mode. The phantom in this benchmark is a $70 \mathrm{~cm}$ long water cylinder with one ${ }^{18} \mathrm{~F}$ (half-life $=109.8 \mathrm{~min}$ ) and one ${ }^{15} \mathrm{O}$ (half-life $=2.03 \mathrm{~min}$ ) line source each with an activity of $100 \mathrm{kBq}$. The simulated acquisition is 4 min in duration, which represents approximately two ${ }^{15} \mathrm{O}$ half-lives. The source activities are set such that the PET benchmark will run in about $12 \mathrm{CPU}$ hours on a $1 \mathrm{GHz}$ processor. The acquisition is divided into two 2 min frames; after the first frame, the gantry rotates by $22.5^{\circ}$. Only coincident events are recorded, using a coincidence time window of $120 \mathrm{~ns}$. This large window is used in order to record a large number of random coincidences. The lower and upper energy thresholds are set to 350 and $650 \mathrm{keV}$, respectively.

The standard electromagnetic package of Geant 4 is used in this benchmark. Only the Rayleigh interactions are modelled using the low energy package. To speed up the simulation, $\mathrm{x}$-rays and secondary electrons are not tracked.

Approximately $3.7 \times 10^{7}$ decays occur during the simulated acquisition and around $7.0 \times 10^{5}$ coincidences are recorded and written in a ROOT file (Brun and Rademakers 1997). Based on the ROOT output, several figures and plots are calculated using ROOT to confirm the correct execution of the simulation. The benchmark results are characterized by: (1) the total number of generated events and detected coincidences; (2) their spatial and time distributions; (3) the fractions of random and scattered coincidences and (4) the average acollinearity between the two annihilation gammas.

The PET benchmark has been run on 12 different system configurations. Two operating systems were tested: Linux and Mac OS (versions 10.2.6 and 10.3). The Linux distributions were RedHat (versions 9.0, 8.0 and 7.3) and SuSE (version 8.1). The source code compilation was performed with either gcc 2.95 or 3.2. The computing time for the PET benchmark averaged around 12 and $6 \mathrm{~h}$ for $1.0 \mathrm{GHz}$ and $3 \mathrm{GHz}$ processors, respectively.

PET physical variables characterizing the simulation results are shown in table 1 with their mean value and standard deviation obtained from the run of the PET benchmark on the 12 system configurations, using a different seed of the random generator on each system. The 


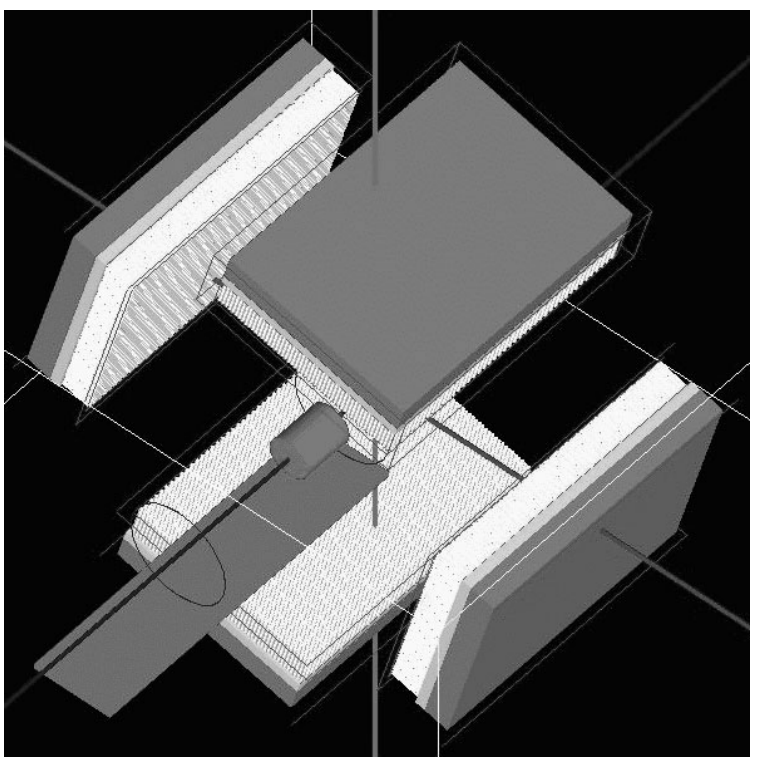

Figure 5. Illustration of the SPECT benchmark set-up.

Table 1. Average values and relative standard deviations (stdev) of the physical variables studied with the PET benchmark.

\begin{tabular}{lll}
\hline Variable type & Average value & Relative stdev (\%) \\
\hline Total decays during the acquisition & $3.6815 \times 10^{7}$ & \pm 0.01 \\
Random coincidences & 20568 & \pm 0.58 \\
Unscattered coincidences & 311778 & \pm 0.39 \\
Scattered coincidences & 369915 & \pm 0.33 \\
Simulated 15-oxygen lifetime & $123.316 \mathrm{~s}$ & \pm 0.06 \\
Gamma acollinearity angle & $0.6063^{\circ}$ & \pm 0.01 \\
\hline
\end{tabular}

results in table 1 and, in particular, the relative standard deviations show that the main physical simulation variables are stable within less than $1 \%$. It is strongly recommended that the user validates a new or updated GATE installation using this table.

\subsection{SPECT benchmark}

The SPECT benchmark (figure 5) simulates a SPECT acquisition with a moving source. The simulated 4-head gamma camera does not correspond to any real system. This benchmark involves a cylindrical phantom (5 cm in diameter and $20 \mathrm{~cm}$ long) filled with water with an inner cylinder ( $2 \mathrm{~cm}$ in diameter, $5 \mathrm{~cm}$ long) filled with $30 \mathrm{kBq}$ of ${ }^{99 m} \mathrm{Tc}$. The phantom lies on a table $(0.6 \mathrm{~cm}$ thick, $8 \mathrm{~cm}$ wide and $34 \mathrm{~cm}$ long). During the simulated acquisition, the table and phantom translate together at $0.04 \mathrm{~cm} \mathrm{~s}^{-1}$. Confinement is used to keep the source distribution synchronized with the phantom movement. All four heads of the gamma camera are identical, consisting of a parallel hole lead collimator (hole diameter: $0.3 \mathrm{~cm}$ collimator thickness: $3 \mathrm{~cm}$ and septa thickness: $0.6 \mathrm{~mm}$ ), a $1 \mathrm{~cm}$ thick NaI crystal, a $2.5 \mathrm{~cm}$ thick back-compartment in Perspex and a $2 \mathrm{~cm}$ thick lead shielding. The simulated acquisition 
Table 2. Average values and relative standard deviations (stdev) of the figures of merit used for the SPECT benchmark.

\begin{tabular}{lcl}
\hline Variable type & Average value & Relative stdev (\%) \\
\hline \multicolumn{4}{c}{ Global information } \\
Number of emitted particles & $1.79994 \times 10^{7}$ & \pm 0.01 \\
Detected counts between 20 and $190 \mathrm{keV}$ & 35919 & \pm 0.3 \\
Percentage of unscattered photons & $32.9 \%$ & \pm 1.0 \\
Mean detected counts per projection & 278.4 & \pm 0.9 \\
\hline Medium & Average value $(\%)$ & Relative stdev (\%) \\
\hline \multicolumn{2}{c}{ Percentage of photons whose last scattered event occurred in a specific medium } \\
Phantom & 52.3 & \pm 0.6 \\
Table & 3.1 & \pm 1.8 \\
Collimator & 2.1 & \pm 2.4 \\
Crystal & 8.5 & \pm 1.2 \\
Back-compartment & 1.2 & \pm 3.3 \\
\hline Scattering order & Average value $(\%)$ & Relative stdev (\%) \\
\hline \multicolumn{1}{c}{ Percentage of scattered photons as a function of the scattering order } \\
Order 1 & 46.4 & \pm 0.7 \\
Order 2 & 26.8 & \pm 0.7 \\
Order 3 & 13.6 & \pm 1.5 \\
Order 4 & 6.7 & \pm 0.7 \\
Order $>4$ & 6.5 & \pm 1.6 \\
\hline
\end{tabular}

consists of 64 projections (16 projections per head), acquired along a circular orbit with a $7 \mathrm{~cm}$ radius of rotation and a speed of $0.15 \mathrm{deg} \mathrm{s}^{-1}$. Sixteen runs of $37.5 \mathrm{~s}$ each are performed to simulate the 16 positions of the 4 gamma camera heads.

The low energy electromagnetic process package is used to model the physics. Rayleigh, photoelectric and Compton interactions are turned on while the gamma conversion interactions are turned off. To speed up the simulation, the x-ray production cut is set at $20 \mathrm{keV}$ and secondary electrons are not tracked. Compton events occurring in the phantom, collimator, back-compartment, shielding and table are recorded. A Gaussian energy blur is applied to all events detected in the crystal, using an energy resolution of $10 \%$ at $140 \mathrm{keV}$. The limited spatial resolution of the photomultipliers and associated electronics is modelled using a Gaussian blur with a standard deviation of $2 \mathrm{~mm}$. Only photons detected with an energy between 20 and $190 \mathrm{keV}$ are stored.

The benchmark results are characterized by: (1) the number of simulated events and of detected counts; (2) the percentage of primary and scattered events with respect to all events detected in the 20-190 keV energy window (here scattered events are considered a function of the compartment in which the last scattered event occurred i.e. phantom, collimator, table, crystal or back-compartment); (3) the mean and standard deviation of the number of detected counts per projection; (4) the percentage of scattered events as a function of the scattering order (first for single scatter, second for double scatter and so on).

The SPECT benchmark has run on 11 system configurations. Two operating systems have been tested: Linux (RedHat versions 9.0, 8.0, 7.3, 7.1, SuSE versions 9.0 and 8.1, and Fedora Core 1) and Mac OS (version 10.2.8). The mean and standard deviation of most figures of merit characterizing the results of the runs are given in table 2. Similar to the PET benchmark results, this table shows that the results produced by GATE are very stable. Table 2 
Table 3. Validation result summary of commercial systems already or currently considered for GATE validation in PET.

\begin{tabular}{|c|c|c|c|}
\hline PET system & Studied FOM & Experiment/GATE & References \\
\hline $\begin{array}{l}\text { ECAT EXACT HR }{ }^{+} \text {, } \\
\text { CPS }\end{array}$ & $\begin{array}{l}\text { Spatial resolution } \\
\text { radial at } 10 \mathrm{~cm} \\
\text { tangential at } 10 \mathrm{~cm} \\
\text { 3D sensitivity } \\
\text { 3D count rates } \\
\text { prompts at } 10 \mathrm{kBq} \mathrm{ml}^{-1} \\
\text { trues at } 10 \mathrm{kBq} \mathrm{ml}^{-1} \\
\text { 3D scatter fraction }\end{array}$ & $\begin{array}{l}11.4 \mathrm{~mm} / 11.8 \mathrm{~mm} \\
11.8 \mathrm{~mm} / 10.7 \mathrm{~mm} \\
0.75 \% / 0.80 \% \\
550 \mathrm{kcps} / 550 \mathrm{kcps} \\
330 \mathrm{kcps} / 300 \mathrm{kcps} \\
36 \% / 35 \%\end{array}$ & Jan et al (2003b) \\
\hline Allegro, Philips & $\begin{array}{l}\text { 3D count rate } \\
\text { trues } \\
\text { scatter } \\
\text { randoms } \\
\text { 3D scatter fraction }\end{array}$ & 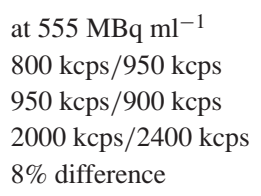 & $\begin{array}{l}\text { Lamare et al } \\
(2004)\end{array}$ \\
\hline GE Advance, GE MS & $\begin{array}{l}\text { Energy spectra } \\
\text { 3D scatter fraction }\end{array}$ & $\begin{array}{l}\text { Visual assessment } \\
47.1 \% / 47.2 \%\end{array}$ & $\begin{array}{l}\text { Schmidtlein et al } \\
\text { (2004) }\end{array}$ \\
\hline $\begin{array}{l}\text { MicroPET } 4, \\
\text { Concorde }\end{array}$ & $\begin{array}{l}\text { Spatial resolution } \\
\text { radial at } 2 \mathrm{~cm} \\
\text { tangential at } 2 \mathrm{~cm} \\
\text { Sensitivity }(350-650 \mathrm{keV}) \\
\text { Miniature Derenzo }\end{array}$ & $\begin{array}{l}2.35 \mathrm{~mm} / 2.25 \mathrm{~mm} \\
2.45 \mathrm{~mm} / 2.30 \mathrm{~mm} \\
1.43 \% / 2.42 \% \\
\text { Visual assessment }\end{array}$ & Jan et al (2003a) \\
\hline $\begin{array}{l}\text { MicroPET Focus, } \\
\text { Concorde }\end{array}$ & $\begin{array}{l}\text { Spatial resolution } \\
\text { radial at } 8 \mathrm{~cm} \\
\text { Sensitivity }\end{array}$ & $\begin{array}{l}3.55 \mathrm{~mm} / 3.4 \mathrm{~mm} \\
3.4 \% / 3.5 \%\end{array}$ & Jan et al (2004) \\
\hline
\end{tabular}

should be used to validate any new installation or update of GATE. The time needed to run the benchmark ranged from about $3 \mathrm{~h}(2.8 \mathrm{GHz}$ Pentium, $2 \mathrm{~Gb}$ RAM $)$ to about $11 \mathrm{~h}(1 \mathrm{GHz}$ Pentium, 2.3 Gb RAM).

\section{Validation of GATE}

The validation of Monte Carlo simulated data against real data obtained with PET and SPECT cameras is essential to assess the accuracy of GATE and the OpenGATE collaboration is largely involved into the validation of GATE. Tables 3 and 4 list commercial systems which have been or are currently being considered for PET and SPECT validations. These tables summarize the figures of merit (FOM) used for assessing the consistency between simulated and real data, as well as the major validation results and associated references. For details regarding these validation studies, the reader is highly encouraged to refer to the appropriate references. Overall, these studies illustrate the flexibility and reliability of GATE for accurately modelling various detector designs. The modelling of the Millennium VG (GE MS) is also in progress.

GATE has also been shown to be appropriate for simulating various prototype imaging devices dedicated to small animal imaging. Table 5 shows the prototypes currently simulated using GATE and indicates the features that have been studied and validated against experimental measurements. 
Table 4. Validation result summary of commercial systems already or currently considered for GATE validation in SPECT.

\begin{tabular}{|c|c|c|c|}
\hline SPECT system & Studied FOM & Experiment/GATE & References \\
\hline IRIX, Philips & None reported & $\mathrm{n} / \mathrm{a}$ & Staelens et al (2004c) \\
\hline AXIS, Philips & $\begin{array}{l}\text { Spatial resolution } \\
\text { Energy spectra } \\
\text { Sensitivity } \\
\text { Scatter profiles }\end{array}$ & $\begin{array}{l}1.30 \mathrm{~cm} / 1.36 \mathrm{~cm} \\
\text { Visual assessment } \\
231 \mathrm{cps} \mathrm{MBq}^{-1} / 246 \mathrm{cps} \mathrm{MBq}^{-1} \\
\text { Visual assessment }\end{array}$ & Staelens et al (2003) \\
\hline Solstice, Philips & Sensitivity & $\begin{array}{l}\text { Good agreement } \\
\text { with theoretical data }\end{array}$ & $\begin{array}{l}\text { Staelens et al }(2003) \\
\text { Staelens et al }(2004 \mathrm{~b}) \\
\text { Staelens et al }(2004 \mathrm{~d})\end{array}$ \\
\hline DST Xli, GEMS & $\begin{array}{l}\text { Energy spectra } \\
\text { Spatial resolution } \\
\text { at } 10 \mathrm{~cm} \text { in air } \\
\text { at } 20 \mathrm{~cm} \text { in water } \\
\text { Sensitivity }\end{array}$ & $\begin{array}{l}\text { Excellent agreement } \\
9.5 \mathrm{~mm} / 9.6 \mathrm{~mm} \\
14.2 \mathrm{~mm} / 14.4 \mathrm{~mm} \\
<4 \% \text { difference }\end{array}$ & Assié et al (2004b) \\
\hline
\end{tabular}

Table 5. Prototypes dedicated to small animal imaging modelled using GATE and features that have been studied using simulated data and summary validation results when available.

\begin{tabular}{|c|c|c|c|}
\hline Prototype & Studied FOM & Experiment/GATE & Reference \\
\hline LSO/LuYAP phoswich PET & $\begin{array}{l}\text { Sensitivity } \\
\text { NEC curves }\end{array}$ & $\begin{array}{l}\mathrm{n} / \mathrm{a} \\
\mathrm{n} / \mathrm{a}\end{array}$ & Rey et al (2003) \\
\hline High resolution dual head PET & $\begin{array}{l}\text { Spatial resolution } \\
\text { at centre } \\
4 \mathrm{~mm} \text { off-centre } \\
\text { Sensitivity } \\
\text { Line phantom }\end{array}$ & $\begin{array}{l}1.60 \mathrm{~mm} / 1.55 \mathrm{~mm} \\
1.72 \mathrm{~mm} / 1.72 \mathrm{~mm} \\
0.13 \% / 0.12 \% \\
\text { Visual assessment }\end{array}$ & Chung et al (2003) \\
\hline CsI(Tl) SPECT camera & $\begin{array}{l}\text { Energy spectra } \\
\text { Spatial resolution } \\
\quad \text { at } 10 \mathrm{~cm} \text { in air } \\
\text { Scatter fraction } \\
\text { Sensitivity } \\
\text { Line phantom }\end{array}$ & $\begin{array}{l}\text { Good agreement } \\
6.7 \mathrm{~mm} / 6.8 \mathrm{~mm} \\
0.531 \% / 0.527 \% \\
<2 \% \text { difference } \\
\text { Visual assessment }\end{array}$ & Lazaro et al (2004) \\
\hline OPET & $\begin{array}{l}\text { Spatial resolution } \\
\text { Sensitivity }\end{array}$ & $\begin{array}{l}\mathrm{n} / \mathrm{a} \\
\mathrm{n} / \mathrm{a}\end{array}$ & Rannou et al (2003) \\
\hline
\end{tabular}

\section{Work in progress and future developments}

\subsection{Speed-up techniques}

Compared to simpler dedicated codes such as SimSET (Harrison et al 1993), the versatility of GATE comes at the expense of relatively long computation times. To compensate for this, variance reduction tools are currently being developed for GATE. Another approach to improve the computing performance of GATE is to distribute the simulations on multiple architectures. This is referred to as the gridification of GATE, and consists of subdividing simulations on geographically distributed processors in a Grid environment by parallelizing the random number generator. GATE simulations use a very long period pseudo-random number generator developed from the algorithm of James (James 1990, Marsaglia and 
Table 6. Comparison of computing times between local and parallel jobs.

\begin{tabular}{lr}
\hline Total computing time in minutes & \\
\hline One $1.5 \mathrm{GHz}$ Pentium IV & 159 \\
10 jobs & 31 \\
20 jobs & 21 \\
50 jobs & 31 \\
100 jobs & 38 \\
\hline
\end{tabular}

Zaman 1987). This random number generator can be subdivided into 900 million different non-overlapping sub-sequences, each containing approximately $10^{30}$ numbers. These subsequences provide convenient starting points throughout the main sequence. Parallel simulations are produced by using the sub-sequences as independent streams by a sequence splitting method (Traore and Hill 2001). To demonstrate the potential benefits of gridification, a simulation was performed. Table 6 gives the total computing time in minutes of a simulation running on a single $1.5 \mathrm{GHz}$ Pentium IV and the same simulation split between 10, 20, 50 and 100 processors on the European DataGrid testbed. In this experiment, the testbed consisted of 200 dual processors with a mix of $750 \mathrm{MHz}$ and $1 \mathrm{GHz}$ Pentium III and $1.4 \mathrm{GHz}$ Pentium IV processors. Gridification using 20 jobs decreased the computing time by a factor of 8 when compared to using a single Pentium IV $1.5 \mathrm{GHz}$ processor. This example emphasizes the fact that computing time is not proportional to the number of jobs running in parallel, due in part to the launch time of the jobs, the modelling of the geometry of the simulation (which is independent of random number generation) and the time spent in queuing jobs. This study also proved that the results obtained via the Grid were equivalent to those generated on a single machine. In the future, each Unix platform on the DataGrid will be installed with GATE, allowing the use of more than 500 processors for a simulation. A convenient tool to split, launch and retrieve GATE simulations on a Grid environment using a Web interface is currently under development.

\subsection{Extension of GATE to other domains}

Besides its application to classical emission tomography, GATE is potentially appropriate to simulate in-line tomography in hadrontherapy. Indeed, GATE possesses the ability to model the distribution of $\beta^{+}$emitters along the beam path. In principle, this distribution can be obtained from knowledge of the nuclear cross-sections of the reactions between heavy ions and target nuclei. This distribution can then be accurately reproduced in GATE using a voxelized source, composed of the different emitters $\left({ }^{15} \mathrm{O},{ }^{11} \mathrm{C}\right.$ and $\left.{ }^{10} \mathrm{C}\right)$.

Currently, Monte Carlo simulations are believed to be the most accurate method for dose calculation in radiotherapy and brachytherapy. A comparison of the computational codes in radiation dosimetry is underway (Berger $e t a l$ ). In this context, the anisotropy function $F(r, \theta)$ (Nath et al 1995) of a ${ }^{192} \mathrm{Ir}$ brachytherapy source in water has been studied. As illustrated in figure 6, anisotropy functions in water have been calculated using Geant3 (Brun et al 1987), MCNP4C (Briesmeister 1993), MCPT (Williamson 1998) and GATE at a radial distance of $2 \mathrm{~cm}$. GATE was in agreement with all other codes. The relative deviation concerning anisotropy functions between MCPT and GATE simulations is less than 3\%. This indicates that GATE has potential to perform dose calculations in brachytherapy. The challenge for GATE is to provide detailed descriptions of local doses delivered by radiotherapy or brachytherapy treatment. It is envisioned that this can be done with voxelized phantoms in future GATE simulations. 


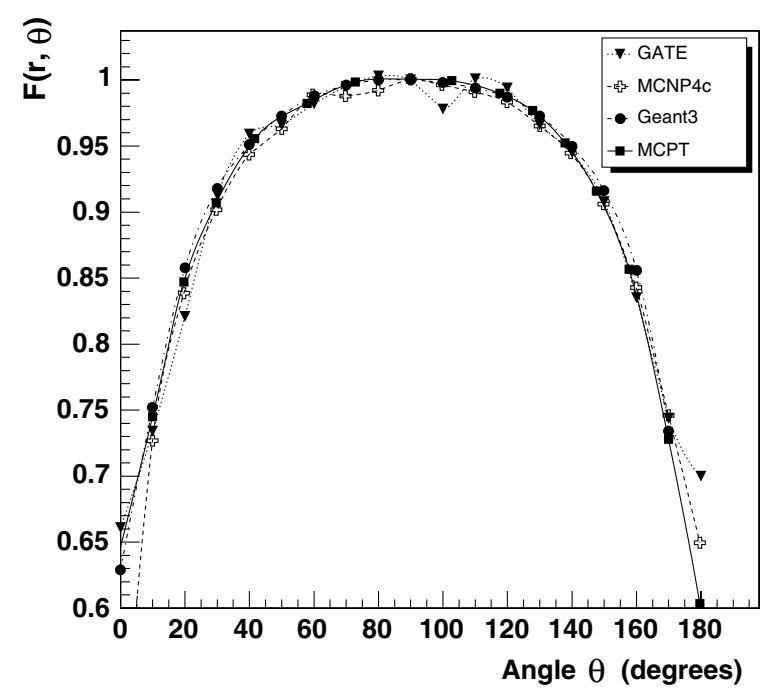

Figure 6. Anisotropy functions at $r=2 \mathrm{~cm}$ for a ${ }^{192} \mathrm{Ir}$ brachytherapy source. The anisotropy functions $F(r, t)$ have been normalized to one at $90^{\circ}$.

\section{Conclusion}

Based on Geant4, GATE is a versatile and adaptable platform for simulating PET and SPECT experiments. GATE is appropriate for simulating conventional scanners and novel detection devices, and does not require any knowledge of $\mathrm{C}++$. The OpenGATE collaboration, representing a large number of research groups from around the world, has publicly released this simulation toolkit after two years of software development and validation. The source code is available for download, which will enable users to make modifications to suit their particular needs. Documentation is available and user support is provided through a very active mailing list.

The future of GATE is closely related to the future of Monte Carlo simulations in nuclear medicine. Because Monte Carlo simulations are playing an increasing role in the optimization of detector design and in the assessment of acquisition and processing protocols, we hope that GATE will answer many of these needs. In addition, the efforts of the OpenGATE collaboration should ensure that GATE will continue to evolve to become a comprehensive simulation tool at the service of the nuclear medicine community.

\section{Acknowledgments}

This work was supported by the Swiss National Science Foundation under grant nos. 2153-063870 and 205320-100472, by the Korea Health 21 R\&D Project under grant no. 02-PJ3-PG6-EV06-0002, by the US National Cancer Institute under grant nos. CA-78573 and CA059017-12, by the Agassiz Foundation, by the Swiss-French Integrated Action Programme Germaine de Staël, by the Institute for the Promotion of Innovation by Science and Technology in Flanders (IWT, Belgium) and by the Fund for Scientific Research in Flanders (FWO, Belgium).

The OpenGATE collaboration is grateful to the Geant4 collaboration, and particularly to the Low Energy Working Group of the Geant4 collaboration for their help and support during 
the starting phase of the development of GATE. In addition, U494 is grateful to the Ligue Nationale Contre la Cancer for supporting Karine Assié's research. The authors also appreciate the support provided by Ghent University and by Philips Medical Systems (INSERM U650).

\section{References}

Agostinelli S et al 2003 GEANT4 - a simulation toolkit Nucl. Instrum. Methods A 506 250-303

Assié K et al 2004a Monte Carlo simulation in PET and SPECT instrumentation using GATE Nucl. Instrum. Methods A 527 180-9

Assié K, Gardin I, Vera P and Buvat I 2004b Validation of Monte Carlo simulations of Indium 111 SPECT using GATE $J$. Nucl. Med. 45 414P-5P

Behrens H and Janecke J 1969 Numerical Tables for Beta-Decay and Electron Capture Landolt-Börnstein, New Series, Group I vol 4 ed H Schopper (Berlin: Springer)

Berger L et al Intercomparison on the Usage of Computational Codes in Radiation Dosimetry, ENEA/QUADOS, http://www.enea.it:/com/ingl/default.htm

Bielajew A F, Hirayama H, Nelson W R and Rogers D W O 1994 History, overview and recent improvements of EGS4 NRCC Report PIRS-0436, National Research Council, Ottawa, Canada

Briesmeister J F (ed) 1993 MCNP-A general Monte Carlo N-particle transport code LANL Report LA-12625-M, Los Alamos National Laboratory, Los Alamos, NM, USA

Brun R, Bruyant F, Maire M, McPherson A C and Zanarini P 1987 GEANT3 Technical Report CERN DD/EE/84-1

Brun R and Rademakers F 1997 ROOT_an object oriented data analysis framework Nucl. Instrum. Methods A 389 $81-6$

Buvat I and Castiglioni I 2002 Monte Carlo simulations in SPET and PET Q. J. Nucl. Med. $4648-61$

Chung Y H, Choi Y, Cho G, Choe Y S, Lee K-H and Kim B-T 2003 Optimization of dual layer phoswich detector consisting of LSO and LuYAP for small animal PET Conf. Rec. IEEE Nucl. Sci. Symp. Med. Imag. Conf. (Portland, OR)

Harrison R L, Vannoy S D, Haynor D R, Gillipsie S B, Kaplan M S and Lewellen T K 1993 Preliminary experience with the photon generator module of a public-domain simulation system for emission tomography Conf. Rec. IEEE Nucl. Sci. Symp. and Med. Imag. Conf. (San Francisco) vol 2 pp 1154-8

Iwata K, Greaves R G and Surko C M $1997 \gamma$-ray spectra from positron annihilation on atoms and molecules Phys. Rev. A 55 3586-604

James F 1990 A review of pseudorandom number generators Comput. Phys. Commun. 60 329-44

Jan S 2002 Simulateur Monte Carlo et caméra à xénon liquide pour la Tomographie à Emission de Positons $P h D$ Thesis Université Joseph Fourier, Grenoble, France

Jan S et al 2003a Monte Carlo Simulation for the ECAT EXACT HR+ system using GATE IEEE Trans. Nucl. Sci. at press

Jan S, Chatziioannou A F, Comtat C, Strul D, Santin G and Trebossen R 2003b Monte Carlo simulation for the microPET P4 system using GATE Conf. Rec. HiRes 2003 (Academy of Molecular Imaging, Madrid) Mol. Imaging Biol. 5138

Jan S, Comtat C, Trebossen R and Syrota A 2004 Monte Carlo simulation of the MicroPET focus for small animal J. Nucl. Med. 45 420P

Kawrakow I and Rogers D W O 2003 The EGSnrc code system: Monte Carlo simulation of electron and photon transport NRCC Report PIRS-701, National Research Council, Ottawa, Canada

Kuntner C, Auffray E, Lecoq P, Pizzolotto C and Schneegans M 2002 Intrinsic energy resolution and light output of the $\mathrm{Lu}_{0.7} \mathrm{Y}_{0.3} \mathrm{AP}: \mathrm{Ce}$ scintillator Nucl. Instrum. Methods A 493 131-6

Lamare F, Turzo A, Bizais Y and Visvikis D 2004 Simulation of the Allegro PET system using GATE Proc. SPIE 5368 890-7

Lazaro D 2003 Validation de la plate-forme de simulation GATE en Tomographie d'Emission Monophotonique et application au développement d'un algorithme de reconstruction 3D complète $P h D$ Thesis Université Blaise Pascal, Clermont Ferrand, France

Lazaro D, Buvat I, Loudos G, Strul D, Santin G, Giokaris N, Donnarieix D, Maigne L, Spanoudaki V, Styliaris S, Staelens S and Breton V 2004 Validation of the GATE Monte Carlo simulation platform for modelling a CsI(Tl) scintillation camera dedicated to small-animal imaging Phys. Med. Biol. 49 271-85

LGPL 1999 GNU Lesser General Public License, Version 2.1, February 1999, Copyright (C) 1991, 1999 Free Software Foundation, Inc., 59 Temple Place, Suite 330, Boston, MA 02111-1307, USA

Marsaglia G and Zaman A 1987 Toward a universal random number generator Florida State University FSU-SCRI$87-50$ 
Nath R, Anderson L L, Luxton G, Weaver K A, Williamson J and Meigooni A S 1995 Dosimetry of interstitial brachytherapy sources: recommendations of the AAPM Radiation Therapy Committee Task Group No. 43 Med. Phys. 22 209-34

Rannou F, Kohli V, Prout D and Chatziioannou A 2004 Investigation of OPET performance using GATE, a Geant4based simulation software IEEE Trans. Nucl. Sci. at press

Rey M, Simon L, Strul D, Vieira J-M and Morel C 2003 Design study of the ClearPET LSO/LuYAP phoswich detector head using GATE Conf. Rec. HiRes 2003 (Academy of Molecular Imaging, Madrid) Mol. Imaging Biol. 5119

Rogers D W O, Faddegon B A, Ding G X, Ma C-M, Wei J and Mackie T R 1995 BEAM: a Monte Carlo code to simulate radiotherapy treatment units Med. Phys. 22 503-24

Santin G, Strul D, Lazaro D, Simon L, Krieguer M, Vieira Martins M, Breton V and Morel C 2003 GATE: A GEANT4-based simulation platform for PET and SPECT integrating movement and time management IEEE Trans. Nucl. Sci. 50 1516-21

Schmidtlein C R, Nehmeh S A, Bidaut L M, Erdi Y E, Humm J L, Amols H I and Kirov A S 2004 Validation of GATE Monte Carlo simulations for the GE Advance PET scanner J. Nucl. Med. 45 409P-10P

Simon L, Strul D, Santin G, Krieguer M and Morel C 2004 Simulation of time curves in small animal PET using GATE Nucl. Instrum. Methods A 527 190-4

Staelens S, D'Asseler Y, Vandenberghe S, Koole M, Lemahieu I and Van de Walle R 2004a A three-dimensional theoretical model incorporating spatial detection uncertainty in continuous detector PET Phys. Med. Biol. 49 2337-50

Staelens S, Koole M, Vandenberghe S, D’Asseler Y, Lemahieu I and Van de Walle R 2004b The geometric transfer function for a slat collimator mounted on a strip detector IEEE Trans. Nucl. Sci. at press

Staelens S, Santin G, Vandenberghe S, Strul D, Koole M, D’Asseler Y, Lemahieu I and Van de Walle R 2004c Transmission imaging with a moving point source: influence of crystal thickness and collimator type IEEE Trans. Nucl. Sci. at press

Staelens S, Strul D, Santin G, Koole M, Vandenberghe S, D’Asseler Y, Lemahieu I and Van de Walle R 2003 Monte Carlo simulations of a scintillation camera using GATE: validation and application modelling Phys. Med. Biol. 48 3021-42

Staelens S, Vandenberghe S, De Beenhouwer J, De Clercq S, D’Asseler Y, Lemahieu I and Van de Walle R 2004d A simulation study comparing the imaging performance of a solid state detector with a rotating slat collimator versus a traditional scintillation camera Proc. SPIE 5372 301-10

Strul D 2001a Preliminary specifications of a Geant4-based framework for nuclear medicine simulations ClearPET Technical Report University of Lausanne, Switzerland

Strul D 2001b Specification of a Geant4-based nuclear medicine simulation framework ClearPET Technical Report University of Lausanne, Switzerland

Strul D, Santin G, Lazaro D, Breton V and Morel C 2003 GATE (GEANT4 Application for Tomographic Emission): a PET/SPECT general-purpose simulation platform Nucl. Phys. B (Proc. Suppl.) C 125 75-9

Traore M and Hill D 2001 The use of random number generation for stochastic distributed simulation: application to ecological modelling Conf. Rec. 13th European Simulation Symposium (Marseille) pp 555-9

von Neumann J 1951 Various techniques in connection with random digits-Monte Carlo methods Nat. Bureau Standards AMS 12, pp 36-8

Williamson J F 1998 Monte Carlo simulation of photon transport phenomena Monte Carlo Simulation in the Radiological Sciences ed R L Morin (Boca Raton FL: CRC Press) pp 53-102

Ziemons K et al 2003 The ClearPET LSO/LuYAP phoswich scanner: a high performance small animal PET system Conf. Rec. IEEE Nucl. Sci. Symp. Med. Imag. Conf. (Portland, OR) 